\title{
Clinical Significance of Atrial Natriuretic Peptide in Human Blood
}

\author{
Tohru Yamaji, Miyuki Ishibashi, Fumimaro Takaku, Fujio Sato*, \\ Kyuzi KamoI*, Hideaki NAKAOKA**, Jun FujII**, \\ Kouichi HaSEgawA*** and Hirotoshi MORII***
}

\begin{abstract}
In an attempt to clarify the clinical significance of atrial natriuretic peptide (ANP) in man, plasma levels of immunoreactive ANP were studied in patients with heart diseases and in those with chronic renal failure. When ANP concentrations in pulmonary arterial plasma were compared with hemodynamic variables in patients with heart diseases who underwent cardiac catheterization, a significant positive correlation was found between plasma ANP levels and mean pulmonary capillary wedge pressure, while plasma ANP levels were not significantly correlated to mean right atrial pressure (MRAP). After the injection of contrast medium, both MRAP and plasma ANP levels increased and a significant positive correlation was observed between two variables. Plasma levels of ANP were elevated in patients with congestive heart failure according to the severity. In addition, patients associated with atrial fibrillation showed significantly higher plasma ANP levels than those on sinus rhythm. In patients with paroxysmal atrial arrhythmias, plasma ANP levels increased markedly during paroxysms. Patients with chronic renal failure had elevated plasma ANP levels, which fell after hemodialysis. These results suggest that both left and right atrial tissue can secrete ANP as a result of stretching of the cardiocytes in man and that plasma ANP levels are elevated in patients with congestive heart failure and in those with chronic renal failure by increased atrial pressure due to volume expansion. Abnormal atrial contraction per se, in addition, may stimulate ANP secretion.
\end{abstract}

Key Words: Plasma ANP levels, Atrial pressure, Congestive heart failure, Chronic renal failure, Atrial arrhythmia

A new peptide with potent natriuretic and vasoactive properties (atrial natriuretic peptide: ANP) has been recently isolated from human atrial tissue, sequenced and synthesized ${ }^{1)}$. A bolus injection of ANP to healthy subjects was shown to cause diuresis, natriuresis and a fall in blood pressure $^{2)}$. A considerable body of evidence is now being accumulated for ANP as a circulating hormone in $\operatorname{man}^{3-11)}$. Plasma levels of ANP are increased by saline infusion ${ }^{3)}$ or increased sodium intake ${ }^{6)}$ suggesting that ANP is secreted in response to volume expansion. If ANP is really a circulating hormone in man, then its plasma levels should be changed in patients with water and electrolyte disorders and in those with altered cardiac function. The present study was undertaken 1) to clarify the mechanism responsible for ANP release in man and 2) to examine the circulating ANP levels in patients with impaired cardiac or renal function.

\section{SUBJECTS AND METHODS}

\section{Subjects}

Experiment 1. Seventeen patients with suspected heart disease (9 men and 8 women), aged

From The Third Department of Internal Medicine, Faculty of Medicine, University of Tokyo, Tokyo, the *Department of Internal Medicine, Nagaoka Red Cross Hospital, Nagaoka,

${ }^{* *}$ Institute for Adult Diseases, Asahi Life Foundation, Tokyo, and

the ***Second Department of Internal Medicine, Osaka City University Medical School, Osaka.

Received for publication November 4, 1986.

Reprint request to: Tohru Yamaji, MD, The Third Department of Internal Medicine, Faculty of Medicine,

University of Tokyo, 7-3-1 Hongo, Bunkyo-ku, Tokyo 113, Japan. 
15-76 years, underwent cardiac catheterization and angiocardiography. Five patients had coronary artery stenosis, seven patients valvular heart diseases, and one patient a small ventricular septal defect. No significant coronary artery stenosis was found in four of these patients.

Experiment 2. Fourteen patients with paroxysmal atrial arrhythmias ( 11 men and 3 women), aged between 35 and 77 , were studied. The types of arrhythmias were atrial fibrillation in $11 \mathrm{pa}$ tients, atrial flutter in two and supraventricular tachycardia in the remaining one.

Experiment 3. Ninety-three patients with heart diseases (56 men and 37 women) were studied. Ages of the patients ranged from 36 to 85 years. Their underlying diseases were valvular heart diseases in 34 patients, myocardial infarction in 18, primary myocardial diseases in 10 , persistent atrial fibrillation without organic heart diseases (lone atrial fibrillation) in 21 and miscellaneous heart diseases in 10.

Experiment 4. Fifty-seven patients with chronic renal failure (CRF) (28 men and 29 women), aged 33-72 years, were studied. The causes of CRF were chronic glomerulonephritis in 44 patients and diabetic nephropathy in the remaining 13 . Thirty-two of the patients were undergoing hemodialysis two or three times a week. The duration of dialysis therapy ranged from 8 to 164 months.

Heparinized blood samples were collected from these subjects on ad libitum water intake. Plasma was separated by centrifugation and stored at $-20^{\circ} \mathrm{C}$ until assayed.

Plasma ANP assay Plasma concentrations of ANP were determined by a specific and sensitive radioimmunoassay after separation of ANP from plasma by means of affinity chromatography on anti-ANP-coupled agarose. The detail of the radioimmunoassay was described previously ${ }^{3)}$. The recovery of ANP from plasma was $80.7 \pm 1.0 \%$ (mean \pm SEM, $\mathrm{n}=37$ ) and the sensitivity of the assay was $12.5 \mathrm{pg} / \mathrm{ml}$. The coefficients of variation averaged $7.2 \%$ for intra-assay error and $11.1 \%$ for inter-assay error. Plasma immunoreactive ANP concentrations in 108 healthy control subjects (54 men and 54 women), aged 21-76 years, ranged from 12.5 to $83.9 \mathrm{pg} / \mathrm{ml}(37.9 \pm 1.4 \mathrm{pg} / \mathrm{ml}$, mean $\pm \mathrm{SEM})$.
Statistical analyses The significance of differences was calculated by Student's $t$ test. The Bonferroni correction was employed for multiple comparisons.

\section{RESULTS}

Experiment 1. Sixteen of the 17 patients studied had normal mean right atrial pressure (MRAP) and mean pulmonary capillary wedge pressure (MPCWP) values, while the remaining patient had slightly increased MRAP and MPCWP. When ANP concentrations in pulmonary arterial plasma were compared with MPCWP, a significant positive correlation $(\mathrm{r}=0.81, \mathrm{p}<0.001)$ was found. There was no significant correlation between plasma ANP levels and MRAP.

After the injection of contrast medium for the purpose of cardioangiography, both MRAP and plasma ANP levels increased. A significant positive correlation was observed between ANP concentrations in pulmonary arterial plasma and MRAP after the injection $(\mathrm{r}=0.62, \mathrm{p}<0.01)$.

Experiment 2. Figure 1 shows changes in plasma ANP levels during paroxysmal atrial arrythmias in 14 patients studied. In all of the patients, plasma ANP levels rose markedly during arrythmias being 290 to $1,470 \%$ of the levels during sinus rhythm. Plasma levels of ANP during sinus rhythm, on the other hand, were normal in

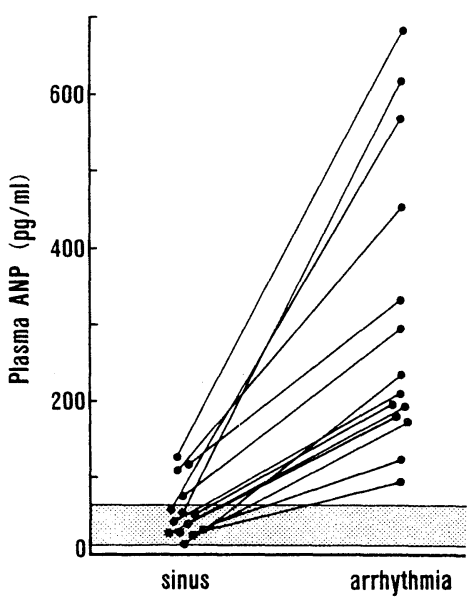

Fig. 1. Changes in plasma ANP levels during paroxysmal atrial arrhythmias in 14 patients. Shaded area represents $95 \%$ confidence limit of plasma ANP levels in healthy subjects. 
11 patients without other cardiac complications, while they were elevated in 3 patients who had organic heart diseases. Symptomatic polyuria was accompanied by paroxysms in 4 of the patients.

Experiment 3. In Figure 2 are shown plasma levels of ANP in patients with various heart diseases including lone atrial fibrillation. Patients were divided according to the severity (New York Heart Association Class: NYHA class) and depending upon the presence or absence of atrial fibrillation. Patients with severe congestive heart failure had higher plasma ANP levels. In addition, patients associated with atrial fibrillation showed significantly higher plasma ANP levels than those on sinus rhythm in NYHA classes I and II. Plasma ANP levels in patients with lone atrial fibrillation $(80.5 \pm 6.1 \mathrm{pg} / \mathrm{ml}$, mean $\pm \mathrm{SEM}, \mathrm{n}=21)$ were significantly $(p<0.01)$ higher than in control subjects.

Experiment 4 . The mean plasma ANP concentration in CRF patients $(173 \pm 17.0 \mathrm{pg} / \mathrm{ml}$, mean \pm SEM, $n=57)$ was significantly $(p<0.01)$ higher than in normal subjects. In addition, predialysis plasma ANP levels in 32 patients on maintenance hemodialysis were significantly $(\mathrm{p}<0.01)$ higher than in 25 non-dialyzed CRF patients $(236 \pm 23.9$ $\mathrm{pg} / \mathrm{ml}$ vs $88.2 \pm 8.9 \mathrm{pg} / \mathrm{ml})$. A significant positive correlation was found between plasma ANP levels and mean blood pressure $(\mathrm{r}=0.59, \mathrm{p}<0.01)$, and plasma ANP levels and cardiothoracic ratios on chest X-ray films $(r=0.70, p<0.01)$ in non-dialyzed patients, while no significant correlation was observed between plasma ANP levels and creatinine concentrations in either group of patients.

Homodialysis resulted in a fall in plasma ANP levels in all 32 patients (Figure 3). The mean ( \pm SEM) plasma ANP concentration significantly ( $p$ $<0.01)$ decreased from predialysis levels $(236 \pm$ $23.9 \mathrm{pg} / \mathrm{ml}$ ) to $100 \pm 12.4 \mathrm{pg} / \mathrm{ml}$. A significant positive correlation was found between $\%$ decrease in plasma ANP concentrations and body weight loss after hemodialysis $(\mathrm{r}=0.43, \mathrm{p}<0.02)$.

In 7 patients with $\mathrm{CRF}$, temporal changes in plasma ANP and serum creatinine levels after hemodialysis were examined. The mean serum creatinine concentrations 24 hours and 48 hours after dialysis were significantly $(\mathrm{p}<0.05$ and $\mathrm{p}<$ 0.01 , respectively) higher than immediately after dialysis. In contrast, plasma ANP levels did not

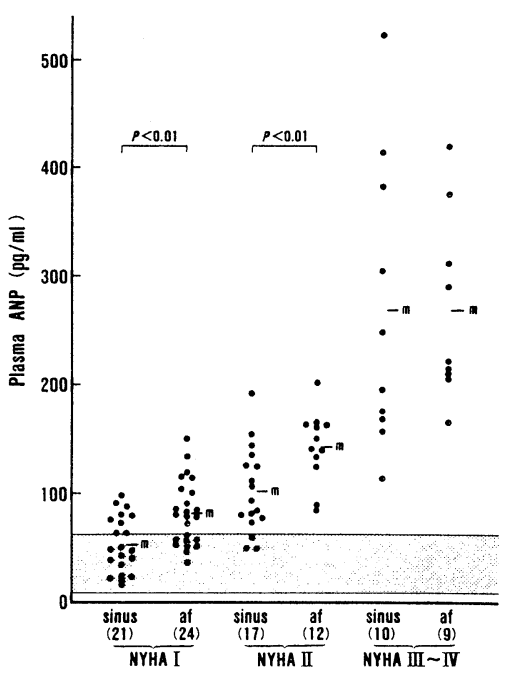

Fig. 2. Plasma ANP levels in patients with heart diseases. Patients were divided according to the functional classification of New York Heart Association (NYHA) and depending upon the presence or absence of atrial fibrillation (af). Shaded area represents $95 \%$ confidence limit of plasma ANP levels in healthy subjects.

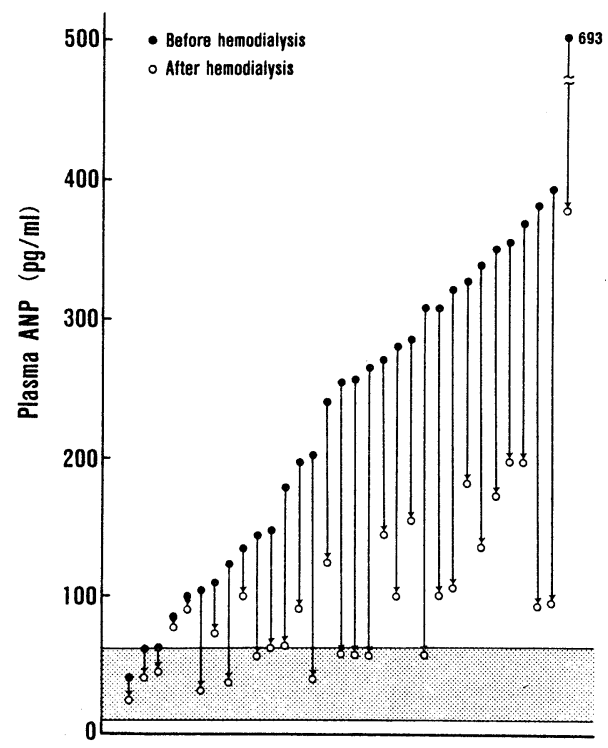

Fig. 3. Changes in plasma ANP levels after hemodialysis in 32 patients with chronic renal failure. Shaded area represents $95 \%$ confidence limit of plasma ANP levels in healthy subjects.

change during the first 24 hours and then rapidly increased during the next 24 hours. 


\section{DISCUSSION}

When ANP concentrations in pulmonary arterial plasma were compared to hemodynamic variables, ANP levels were positively correlated to MPCWP, while no significant correlation was observed between plasma ANP levels and MRAP. Since MPCWP reflects mean left atrial pressure, it appears that plasma ANP concentrations are positively correlated to mean left atrial pressure. Thus, left atrial pressure may play an important role in ANP release when right atrial pressure is not elevated. Injection of contrast medium elevated both MRAP and plasma ANP levels, and a significant positive correlation was obtained between plasma ANP levels and MRAP. The increased MRAP after injection of contrast medium may be due to increased volume loading or depressed cardiac function $^{12,13)}$. The result suggests that an increase in right atrial pressure also triggers ANP release. This view may be supported by the observation by Lang et $a l .{ }^{14)}$, who found a correlation between plasma levels of atrial natriuretic factor and MRAP in rats when MRAP was increased by isotonic saline infusion. It was concluded from these results that both atrial tissue can secrete ANP in response to an increase in pressure in man.

The present study clearly shows that plasma ANP levels are consistently elevated during paroxysmal atrial arrhythmias. The levels during arrhythmias were several times as high as those during sinus rhythm in most patients. It is well known that paroxysmal atrial arrhythmias are often accompanied by polyuria ${ }^{15)}$. This phenomenon has been attributed to transient suppression of arginine vasopressin (AVP) secretion from the neurohypophysis via atrial distension ${ }^{15,16)}$. However, natriuresis during paroxysms could not be explained simply by the inhibition of AVP release. Thus, ANP may be at least one of the main factors involved in diuresis and natriuresis accompanied by paroxysmal atrial arrhythmias.

Plasma ANP levels were elevated in patients with congestive heart failure, which is consistent with previous reports by us and others ${ }^{5-8}$ ). In addition, patients associated with atrial fibrillation had significantly higher plasma ANP levels when compared to those on sinus rhythm in the same NYHA class. Plasma ANP levels in patients with lone atrial fibrillation were again statistically higher than in control subjects. Since an increase in atrial pressure has not been demonstrated in patients with lone atrial fibrillation ${ }^{17}{ }^{18}$ ), ANP secretion may be stimulated by abnormal atrial contraction with or without increased atrial pressure.

Plasma levels of ANP were elevated in patients with CRF. In normal subjects, plasma ANP levels increase in response to saline infusion ${ }^{3)}$, are suppressed by low sodium intake, and correlate with urinary sodium excretion ${ }^{6}$. In view of these findings, expanded extracellular fluid volume in CRF is probably responsible for elevated plasma ANP levels in CRF patients. Another possible explanation for elevated plasma ANP in CRF patients may be impaired metabolism and excretion of ANP by the non-functioning kidneys. In fact, specific membrane receptors for atrial natriuretic factor were demonstrated in rat renal cortex ${ }^{19)}$. Tang et $a l .{ }^{20)}$ reported that iodinated atriopeptin III was rapidly cleared from the circulation in rats with a biological half-life of $2.5 \mathrm{~min}$, and suggested that the kidney is the main organ where the ANP degradation may take place. However, the lack of significant correlation between plasma ANP and serum creatinine concentrations in both dialyzed and non-dialyzed patients argues against this possibility. Moreover, the temporal relationship between plasma ANP and serum creatinine levels after hemodialysis indicates that the main cause for the elevated plasma ANP levels in CRF patients may be increased ANP secretion due to volume expansion rather than decreased ANP clearance.

Hemodialysis consistently diminished plasma ANP levels and the \% decrease in plasma ANP after hemodialysis correlated significantly with body weight loss during hemodialysis. The results again support the view alluded to above that high plasma ANP levels in CRF may be attributed to an increase in extracellular volume. Recently, Rascher et $a l .{ }^{9)}$ reported, though in a limited number of patients, that hemodialysis decreased plasma ANP levels in children with advanced renal failure. Of note in this regard is that reduction in plasma ANP levels may not be solely due to decreased ANP secretion. In fact, a small but significant amount of ANP was detected in dialysis fluid (unpublished observation) suggesting that ANP was removed in 
part by filtration through the dialysis membrane.

In conclusion, the present study demonstrates that, in man, increased atrial pressure may be an important factor that induces ANP release. Both left and right atria may secrete ANP, as a result of stretching of the cardiocytes secondary to increased atrial pressure. Elevated plasma ANP levels in patients with congestive heart failure and in those with CRF may be explained by an increase in atrial pressure due to volume expansion. Abnormal atrial contraction per se, in addition, may stimulate ANP secretion.

\section{REFERENCES}

1) Kangawa $\mathrm{K}$, Matsuo $\mathrm{H}$ : Purification and complete amino acid sequence of $\alpha$-human atrial natriuretic polypeptide. Biochem Biophys Res Commun 118: $131,1984$.

2) Richards AM, Ikram H, Yandle TH, et al: Renal, haemodynamic and hormonal effects of human alpha atrial natriuretic peptide in healthy volunteers. Lancet i: 545, 1985.

3) Yamaji $T$, Ishibashi M, Takaku F: Atrial natriuretic factor in human blood. J Clin Invest 76: 1705, 1985.

4) Yamaji $T$, Ishibashi $M$, Nakaoka $H$, et al: Possible role for atrial natriuretic peptide in polyuria associated with paroxysma atrial arrhythmias. Lancet $\mathbf{i}$ : $1211,1985$.

5) Nakaoka H, Imataka $\mathrm{K}$, Amano $\mathrm{M}$, et al: Plasma levels of atrial natriuretic factor in patient with congestive heart failure. N Engl J Med 313: 892, 1985.

6) Shenker Y, Sider RS, Ostafin EA, et al: Plasma levels of immunoreactive atrial natriuretic factor in healthy subjects and in patients with edema. J Clin Invest $76: 1684,1985$.

7) Tikkanen I, Fyhrquist F, Metsärinne K, et al: Plasma atrial natriuretic peptide in cardiac disease and during infusion in healthy volunteers. Lancet ii: 66, 1985.

8) Hartter E, Weissel M, Stummvoll HK, et al: Atrial natriuretic peptide concentrations in blood from right atrium in ptients with severe right heart failure. Lancet ii: 93, 1985.

9) Rascher W, Tulassay T, Lang RE: Atrial natriuretic peptide in plasma of volume-overloaded children with chronic renal failure. Lancet ii: 303, 1985.

10) Gutkowska J, Bourassa M, Roy D, et al: Immunoreactive atrial natriuretic factor in human plasma. Biochem Biophys Res Commun 128: 1350, 1985.

11) Kimura $T$, Abe $K$, Ota $K$, et al: Effects of acute water load, hypertonic saline infusion, and furosemide administration on atrial natriuretic peptide and vasopressin release in humans. J Clin Endocrinol Metab 62: 1003, 1986.

12) Brown R, Rahimtoola SH, Davis GD, et al: The effect of angiocardiographic contrast medium on circulatory dynamics in man: cardiac output during angiocardiography. Circulation 31: 234, 1965.

13) Gootman N, Rudolph AM, Buckley NM: Effects of angiographic contrast media on cardiac function. Am J Cardiol 25: 59, 1970.

14) Lang RE, Thölken H, Ganten D, et al: Atrial natriuretic factor-a circulating hormone stimulated by volume loading. Nature (London) 314: 264, 1985.

15) Wood P: Polyuria in paroxysmal tachycardia and paroxysmal atrial flutter and fibrillation. Br Heart J 25: 273, 1963.

16) Canepa-Anson R, Williams M, Marshall J, et al: Mechanism of polyuria and natriuresis in atrioventricular nodal tachycardia. Br Med J 289: 866, 1984.

17) Khaja F, Parker JO: Hemodynamic effects of cardioversion in chronic atrial fibrillation. Arch Intern Med 129: 433, 1972.

18) Orlando JR, van Herick R, Aronow WS, et al: Hemodynamics and echocardiograms before and after cardioversion of atrial fibrillation to normal sinus rhythm. Chest 76: 521, 1979.

19) Napier MA, Vandlen RL, Albers-Schönberg G, et al: Specific membrane receptors for atrial natriuretic factor in renal and vascular tissues. Proc Natl Acad Sci USA 81: 5946, 1984.

20) Tang J, Webber RJ, Chang D, et al: Depressor and natriuretic activities of several atrial peptides. Regul Peptide 9: 53, 1984. 